\title{
Not Compounding from Bulk Ingredient
}

National Cancer Institute

\section{Source}

National Cancer Institute. Not Compounding from Bulk Ingredient. NCI Thesaurus. Code C112093.

A compounder produces a drug without bulk ingredients. 